\title{
Culinary traditions-revealing the effect of travel and diet on gut microbiota and motility
}

A ccording to new research published in Cell, short-term changes in diet can regulate gut motility. This effect correlated with changes in the levels of unconjugated bile acids. Turmeric, a spice prevalent in Bangledeshi cuisine, was shown to affect gut motility via cholekinesis, which was modulated by bacterial bile salt hydrolase (BSH) activity and mediated by Ret signalling in the enteric nervous system (ENS).

"At present, we lack detailed understanding of the complex and dynamic interrelationships between the gut microbiota, the ENS and host genetics," says corresponding author Jeffrey Gordon. "Our understanding is particularly lacking in the global context of diverse cultural traditions concerning foods, their methods of preparation and the varied human gut microbiota that have evolved under these dietary conditions."

To study these relationships the authors transplanted faecal microbiota from human donors representing different culinary traditions into gnotobiotic mice (which have a known gut microbial community). To mimic the changes in diet experienced by us when we travel, the mice were fed a diet representative of each destination for 8 days. The starting and ending diet were those of the 'local' diet and were given for 14 days and 8 days, respectively. The donors' country of origin and corresponding traditional diets were as follows: USA with an unrestricted diet (USA-U); USA with a diet rich in fat and protein (USA-P); rural Venezuela; rural Malawi; urban Bangladesh.

Diet, donor microbiota and the interaction between the two were found to be statistically significant factors affecting faecal transit time. The quickest transit times were seen in mice with contrasting diet and microbiota combinations: mice colonized with USA-U microbiota and fed the Bangladeshi diet, and mice with Bangladeshi microbiota fed the USA-P diet. In mice with these contrasting microbiota and diets, 27 operational taxonomic units (OTUs) had dietdependent correlations with transit time. The effect of bacteria on transit time was not always universal: for example, when fed the USA-P diet two strains of Eubacterium desmolans had opposing effects on motility in mice colonized with Bangladeshi microbiota.

Transit times during the initial and final 'local' diets were often different and changes in the abundance of some bacteria occurred. The researchers looked at the metabolic profile of the microbiota communities to see if they might alter transit times. Massspectrometry analysis of metabolites in mouse faeces found hundreds of peaks associated with transit times and 67 (11\%) were products of bile acid metabolism. Gut bacteria metabolize bile acids, initially by deconjugating them with BSHs to reduce their microbicidal activity. Unconjugated bile acids correlated with faster transit times, whereas the reverse was observed with conjugated bile acids.

To study relationships between diet, microbiota, motility and host genetics the authors chose to focus on turmeric, as the active ingredient, curcumin, stimulates the gall bladder and increases bile acid secretion. Mice consuming turmeric had slower transit times compared with those mice that had not consumed the spice.

Two groups of seven bacterial species, with either low or high levels of BSHs $\left(\mathrm{BSH}_{10}\right.$ and $\left.\mathrm{BSH}_{\mathrm{hi}}\right)$, were cultured and mice were colonized with either $\mathrm{BSH}_{\mathrm{lo}}$ or $\mathrm{BSH}_{\text {hi }}$; however, transit time was unaffected and the same in both groups. With the addition of turmeric to their diet, transit times were slower in the $\mathrm{BSH}_{10}$ group (no change was observed in the $\mathrm{BSH}_{\mathrm{hi}}$ group) and genes involved in gut mucosal immunity and barrier function were increased in the terminal ileum.

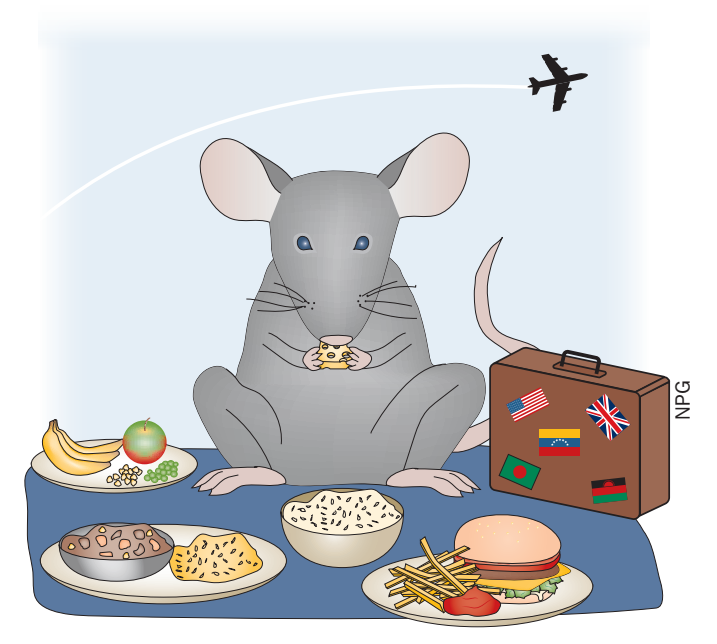

Finally, ENS involvement in mediating the effect of turmeric on motility was studied in $\mathrm{Ret}^{+/-}$mice, which have a dysfunctional ENS. Wild-type mice had lower transit times than $\mathrm{Ret}^{+/-}$mice when both groups were conventionally raised; in gnotobiotic wild-type and $\mathrm{Ret}^{+/-}$mice fed turmeric, this difference was seen in mice colonized with the $\mathrm{BSH}_{\text {hi }}$ but not the $\mathrm{BSH}_{\text {lo }}$ microbiota, suggesting that unconjugated bile acids are key mediators. In addition, the motility difference between $\mathrm{BSH}_{\mathrm{lo}}$ and $\mathrm{BSH}_{\mathrm{hi}}$ wild-type mice that received turmeric in their diet was not seen in $\mathrm{BSH}_{\mathrm{lo}}$ and $\mathrm{BSH}_{\mathrm{hi}}$ Ret $^{+/-}$mice, indicating that a functional ENS and bile acid metabolism is required for the effect turmeric has on gut motility.

"In an era of globalization with rapid disappearance of cultural traditions, these results demonstrate a way for identifying how traditional food ingredients interact with specific functional traits of the human microbiota to regulated host biology," concludes Gordon.

Gillian Patman

\footnotetext{
Original article Dey, N. et al. Regulators of gut motility revealed by a gnotobiotic model of diet-microbiome interactions related to travel. Cell doi:10.1016/ j.cell.2015.08.059
} 